ORIGINAL ARTICLE

\title{
No evidence of an association between MMR vaccine and gait disturbance
}

\author{
E Miller, N Andrews, A Grant, J Stowe, B Taylor
}

Arch Dis Child 2005;90:292-296. doi: 10.1136/adc.2003.048165

\begin{abstract}
See end of article for authors' affiliations

.....................

Correspondence to: Dr E Miller, Immunisation Division, Public Health Laboratory Service, Health Protection Agency, 61 Colindale Avenue, London NW9 5EQ, UK; liz.miller@ hpa.org.uk
\end{abstract}

Accepted 19 May 2004

\begin{abstract}
Background: MMR vaccine has been reported to cause gait disturbance, and this possible association has been claimed to support the MMR-causes-autism theory.

Aims: To determine whether any association between gait disturbance and MMR vaccination exceeds the age related background rate of gait disturbance, using record linkage and self control case series analyses.

Methods: MMR vaccination records were linked to hospital admission and general practitioner attendance data. An increased rate of gait problems with onset in various intervals in the 60 day period after MMR vaccination was looked for in children aged 12 to $<24$ months.

Results: No evidence of an increased rate of hospital admission or general practice consultations for gait disturbance was found in the putative post-vaccination risk periods.

Conclusions: This study provides no evidence for a causal association between MMR and gait disturbance.
\end{abstract}

l n 1995, Plesner reported 24 cases of temporary gait disturbance, in previously normal 15 month old children, shortly after measles/mumps/rubella (MMR) vaccine). ${ }^{1}$ The cases were reported to the statutory adverse event notification system in Denmark between 1987 and 1995 at an incidence of 6 per 100000 doses. The close temporal association with MMR vaccine (range of onset 3-25 days), combined with the failure to find an alternative aetiology, was taken as evidence of a causal association. However, without information on the background incidence of gait disturbance in children aged 12 to $<24$ months, any causal association with MMR must remain speculative.

The Public Health Laboratory Service in England and Wales has developed an efficient and powerful method for investigating adverse vaccine associated events based on linkage of computerised clinical records with vaccination records and use of the self controlled case series (SCCS) method for data analysis. ${ }^{2}$ The SCCS method allows the risk of events in specified post-vaccination periods to be compared with the age specific background risk, without the need for age matched controls yet retaining the power of a cohort study. ${ }^{3}$

We investigated the hypothesis that there is an increased risk of gait disturbance within 60 days of MMR vaccination using computerised hospital admission records linked with immunisation records for children in the Thames region of England for the period April 1995 to June 2001. Because gait disturbance may not result in hospital admission, we also tested the same hypothesis using the General Practice Research Database (GPRD) which holds computerised information on all significant patient consultations, referrals, and prescribed medicines including vaccines from 1988, from around 500 general practices in the UK. Together these practices provide primary health care for 3.4 million patients ( $5.7 \%$ of the population).

Because of recent speculation that exposure to mercury, through the preservative thiomersal in inactivated vaccines given in the first year of life, may "sensitise" a child for subsequent MMR related neurological or developmental problems, ${ }^{4}$ the GPRD data set was also used to test the hypothesis that prior thiomersal exposure in combination with MMR vaccine is a risk factor for subsequent gait disturbance.

\section{METHODS}

Computerised hospital admission and immunisation records for children in the former North and South Thames regions were obtained for the period April 1995 to June 2001 and linked on National Health Service (NHS) number, or sex, date of birth, and full post code-a highly specific linking algorithm. ${ }^{5}$ Based on the annual birth cohort in the districts from which hospital admissions records were obtained, it is estimated that approximately 509000 children were eligible to receive MMR vaccine during the study period. Admissions in children aged 12 to $<24$ months with an ICD-10 diagnosis code indicating a possible acute gait disorder or other condition suggestive of cerebellar dysfunction or disturbed motor control were identified, irrespective of whether a linked MMR record was found. The ICD codes used were G111, G112, G25, R26, R27, R29, H55, and F984.

The ICD 10 diagnosis codes were validated by hospital case note review. Cases were grouped into five categories as follows: (1) presumptive viral/post-viral ataxia (clinical history of ataxia and evidence of encephalomyelitis or cerebellitis with lymphocytosis in the cerebrospinal fluid (CSF) or encephalographic changes); (2) probable post-viral ataxia (history consistent with ataxia but CSF/other investigations inconclusive or not done and no other cause identified); (3) probably not post-viral gait disturbance (vague symptoms not suggestive of cerebellar ataxia, e.g. unsteady gait associated with constipation or gastroenteritis); (4) non-ataxic, non-viral gait disturbance (including limp after trauma, septic bone or joint disease, unsteadiness following drug ingestion); and (5) transient synovitis/ "irritable hip" (a transient condition described following viral illnesses and with no long term sequelae). Categorisation of cases was carried out without knowledge of MMR vaccination status by BT. Vaccination histories for

Abbreviations: CSF, cerebrospinal fluid; DT, diphtheria/tetanus; DTP, diphtheria/tetanus/pertussis; GPRD, General Practice Research Database; MMR, measles/mumps/rubella; SCCS, self controlled case series 
children without a linked immunisation record were checked against the computer records held in child health departments in the study areas.

For the analysis of gait disorders presenting in general practice, information on all children born from 1988 to 1997 with at least two years of continuous follow up from birth in a GPRD practice deemed as supplying data of research standard was obtained from the Office for National Statistics. Data were available up until the end of 1999 in linked patient, medical, and prevention databases for 152898 children, of whom $138190(90.3 \%)$ had a date of MMR recorded.

Read and OXMIS codes that indicated a consultation for possible gait disturbance in children aged 12 to $<24$ months were identified by mapping to ICD-9 codes and by searching on the following keywords: ataxia, gait, co-ordination, mobility, movement. Read/OXMIS descriptive diagnoses cover a wide range, so were grouped into six categories for analysis: (A) ataxia (including cerebellar ataxia and ataxic gait); (B) unsteady/veering/shuffling gait; (C) gait abnormality—unspecified; (D) limp/limping gait; (E) poor mobility; and (F) abnormal /involuntary movements. It had been hoped to match GPRD with hospital categories, but limitations to the GPRD validation (see results) meant that these broad descriptive categories, which largely reflected presenting complaints, had to be used. GPRD category A is the most likely to match hospital categories 1 and 2-those biologically plausible as being vaccination related-but is likely to include a rather broader spectrum of disorder, not necessarily all acute. GPRD category B is also possibly related, although the details of those gait problems were usually unclear. Vaccination histories were obtained from the linked prescription records. A subset of 100 records was selected for validation; these comprised all 12 consultations occurring on the day of vaccination, and additionally 19 coded as ataxia, 51 coded as unsteady gait, 12 coded as abnormal gait, and 6 coded as limping gait.

Post-vaccination risk periods of $0-30$ and 31-60 days after vaccination were specified a priori after discussion with AM Plesner. The incidence of hospital admission or GP consultation for a gait disorder in these risk periods relative to the background rate was measured using the self controlled case series analysis method, ${ }^{3}$ with age stratification in one month intervals. In the GPRD data set, an additional analysis was performed in which the relative incidence (RI) measures were recalculated with stratification according to the level of thiomersal exposure. In the UK, the only thiomersal containing vaccines that are given routinely in the first year of life are diphtheria/tetanus/pertussis (DTP) or DT vaccines, each dose containing $50 \mu \mathrm{g}$ thiomersal ( $25 \mu \mathrm{g}$ mercury). Since 1990 an accelerated 2, 3, 4 month primary immunisation schedule has been used. The maximum mercury exposure for vaccines routinely recommended under the UK infant immunisation schedule is therefore $75 \mu \mathrm{g}$ by 4 months of age. Thiomersal is also present in hepatitis B and influenza vaccines, which in the UK are recommended only for certain high risk children. ${ }^{6}$ No child with suspected gait disturbance had received either of these two vaccines. If mercury is damaging, there should be a higher risk with earlier and higher dosage. For the analysis, the level of thiomersal exposure was defined in two ways. Firstly according to the number of DTP/DT doses received by 4 months of age, and secondly according to the sum across all three doses of the number of days each dose was given prior to 6 months of age (note that any dose not given, or given after 6 months contributed 0 days to this calculation). The latter variable, called mercury exposure intensity, captures the age specific exposure of all three doses and has higher values the earlier the three doses were given. For the analysis, mercury exposure intensity was grouped into quartiles. Since only month/year of birth is routinely provided to researchers using the GPRD, the exact date of birth was derived where possible by identifying the date of any event in the associated medical record that would have occurred on the day of birth; for example, date of APGAR score. ${ }^{7}$ Where this was not possible, date of birth was taken as the 16th day of the month.

\section{RESULTS}

\section{Hospital admissions}

A total of 146 admissions for a possible gait disturbance were identified in children aged 12 to $<24$ months for whom case notes and immunisation records were traced for 127 (87\%). Of these, 114 (90\%) had received MMR vaccine. Non-ataxic, non-viral gait disturbance (category 4 ) was identified as the cause for admission in 65 of the 127 episodes, leaving 62 episodes for analysis, none of which met the category 1 definition. These 62 cases comprised 19 probable post-viral ataxias (category 2), 19 probably not post-viral ataxias (category 3), and 24 gait disturbances considered to be probable "post-viral" transient synovitis (category 5). Table 1 shows the RI estimates for admission in the predefined postvaccination risk periods for the different categories of gait disturbance, excluding category 4 . There was no evidence of an increased RI for any of the categories. For probable postviral ataxia, the category most closely resembling the cases described by Plesner, ${ }^{1}$ the observed RI in the putative postvaccination risk period was 1.13 , based on a total of two cases admitted within 60 days of vaccination, at 22 and 34 days respectively after MMR.

\section{General practice consultations}

A total of 1403 children (1091 in the group with an exact date of birth and 312 in the group for which the day of birth was assigned to the 16th day of the month) aged 12 to $<24$ months presenting with a possible gait disturbance, and in whom the date of the consultation preceded the last date when the practice data were deemed to be "up to research standard", were identified using the selected Read and OXMIS codes. Of these, 24 had a code reflecting some form of ataxia (category A), and 79 had a code denoting unsteady gait (category B); 654 (47\%) had a code denoting an unspecified gait abnormality (category C) and 636 (45\%) had a code where the text description included the terms limp/ing [walk/gait] (category D). The remainder comprised poor mobility (category $\mathrm{E}, \mathrm{n}=7$ ) and abnormal movements (category F, $\mathrm{n}=3$ ). Five of these 1403 children had implausible MMR dates (for example, MMR given early in the first year of life) and were dropped from the main analysis. A further 20 children had implausible DTP dates, so were not included in the secondary analysis that used exposure to thiomersal containing vaccines in the first year of life.

The initial analysis gave a significantly increased RI for the 30 day post-vaccination period for some categories of gait disturbance (B, unsteady; and $\mathrm{C}$, unspecified) that was caused by a clear excess of consultations on the day that MMR was given. It is biologically implausible that any specific MMR effect would be manifest on the day of vaccination since the viraemia induced by the vaccine, which might produce symptoms, does not start until the end of the first week. ${ }^{8}$ Opportunistic vaccination when children presented with coincidental gait disturbance, or expression of concerns about such symptoms when parents presented their child for immunisation, seem more likely explanations. Hence for the subsequent category specific analyses, the 0-30 day post-vaccination period was subdivided into a 0-5 day and 6-30 day period (table 2). 
Table 1 Relative incidence (RI) and $95 \%$ confidence intervals (CI) of hospital admission for possible gait disorder within 60 days of MMR vaccine in children aged 12 to $<24$ months

\begin{tabular}{llll}
\hline Type of gait disturbance & Risk period (days) & RI (95\% CI) & Cases in risk period \\
\hline Any (categories 2, 3, 5; $\mathrm{n}=62$ ) & $0-30$ & $0.83(0.24$ to 2.84) & 3 \\
& $31-60$ & $0.20(0.03$ to 1.47$)$ & 1 \\
Probable post-viral ataxia (category 2; $\mathrm{n}=19$ ) & $0-60$ & $0.46(0.16$ to 1.35$)$ & 4 \\
Probably not post-viral ataxia (category 3; $\mathrm{n}=19)$ & $0-60$ & $1.13(0.21$ to 6.20$)$ & 2 \\
Transient synovitis/irritable hip (category $5 ; \mathrm{n}=24)$ & $0-60$ & 0 (not estimable) & 0 \\
\hline
\end{tabular}

Table 2 shows the results according to type of gait disturbance and post-vaccination risk period. With the exception of the increased incidence of consultation for any gait disturbance $0-5$ days after MMR vaccination (1.88, 95\% CI 1.30 to 2.72 ), attributable to an excess in categories B and $\mathrm{C}$ on the day of vaccination ( $12 / 31$ of all events in the $0-5$ day period), no other post-vaccination risk period showed an increased incidence.

In order to obtain a more precise estimate for the risk of ataxia after MMR, a combined analysis with category 2 from the hospital admissions data and category A from the general practice data was performed. This gave a relative incidence of 0.81 (95\% CI 0.18 to 3.69) for the 0-30 day period and 0.96 (95\% CI 0.33 to 2.82 ) for the $0-60$ day period.

A total of 1378 children were included in the additional analyses involving both MMR and DTP/DT (that is, thiomersal) exposure; of these 60 were recorded as receiving no doses, 26 one dose, 34 two doses, and 1263 (91.6\%) three doses of a DTP/DT vaccine, of whom 476 did so by 4 months of age. These additional analyses showed no evidence of a predictive association between early administration of thiomersal containing DTP/DT vaccines and an MMR associated gait disturbance (table 3 ). This analysis was also performed after excluding the cohort without an exact date of birth available and produced very similar results (for example, RI 6-60 days post-MMR vaccination for categories A-F was 1.06 for those with three DTP doses by 4 months of age and 0.79 for those with three doses after 4 months of age).

Of the 100 cases selected for validation, adequate clinical information was obtained on only 37 (37\%). The reasons for non-validation of the remaining 63 cases were as follows: in 54, the practices no longer participated in the GPRD, five children had transferred out of a participating practice so notes were no longer available, in one the GP did not respond to the validation request, and in a further three no additional information could be found in the notes which related to the consultation for possible gait disturbance.

Five of the 12 events that occurred on the day of MMR vaccination were validated; in three of these the gait disturbance was volunteered when the patient attended for vaccination, in one the patient was opportunistically vaccinated when the patient consulted for gait disturbance, and in one the date of consultation for a hip lesion was wrongly recorded as the date of MMR vaccination one week earlier. Of the 18 cases coded as "gait abnormal" or "limping", nine were validated, all of whom had a traumatic or orthopaedic aetiology. Of the remaining 70 consultations coded as ataxia or unsteady gait for which validation was attempted, information was obtained on 23. Of these, 11 had no problem reported on examination, in five a transient gait disturbance or lack of coordination of uncertain aetiology was diagnosed, in three the gait disturbance was associated with trauma or orthopaedic problem, in two with an infection, in one with epilepsy, and in one with congenital cerebellar disease.

\section{DISCUSSION}

This study provides no evidence that MMR vaccine causes acute ataxia or other gait disturbance and suggests that the cases originally described by Plesner ${ }^{1}$ were chance occurrences, reflecting background incidence. We identified similar rare cases of transient ataxia without an obvious underlying pathology in children aged 12 to $<24$ months, but the incidence was no higher in the two months after MMR vaccine than at other times during the second year of life. Although the cases described by Plesner had onset within 3-25 days of MMR, we specified a longer post-vaccination risk period to allow for possible delays from onset to presentation, in general practice or hospital. The risk based on the cases reported to Plesner (6 per 100000 doses) suggested that the hospital based surveillance, which covered a population of over half a million children aged 12 to $<24$ months, of whom $85 \%$ received MMR vaccine, 9 should have had sufficient power to identify such cases. However, children with acute ataxia or other gait disturbance may not necessarily be admitted to hospital, hence the importance of the GP based study. In the latter, 138190 doses of MMR were given, but no evidence of an excess of consultations was found for ataxia/unsteady gait within 6-60 days of MMR vaccination.

The clinical codes used for identification of episodes that might indicate the type of gait disturbance reported by Plesner $^{1}$ were chosen to maximise sensitivity (that is, minimise the number of true cases missed) rather than specificity (that is, minimise the number of non-cases identified). This is reflected in the high proportion of cases in both the hospital admission and the GP consultation group

Table 2 Relative incidence (and 95\% confidence intervals) of a general practice consultation for possible gait disorder within 60 days of MMR vaccine in children aged 12 to $<24$ months according to type of gait disturbance

\begin{tabular}{|c|c|c|c|c|}
\hline \multirow[b]{2}{*}{ Type of gait disorder (category) } & \multicolumn{4}{|c|}{ Gap between MMR and event date (days) } \\
\hline & $0-5$ & $6-30$ & $31-60$ & $6-60$ \\
\hline All cases (A to $F)$ & $1.88(1.30$ to 2.72$)[31]$ & $0.90(0.70$ to 1.17$)[69]$ & $0.95(0.77$ to 1.19$)$ [102] & $0.93(0.78$ to 1.12$)[171]$ \\
\hline Ataxia $(A)$ & $0(-)[0]$ & $0.72(0.08$ to 6.30$)[1]$ & $1.14(0.21$ to 6.31$)[2]$ & $0.95(0.22$ to 4.12$)$ [3] \\
\hline Unsteady gait (B) & $6.90(2.67$ to 17.8$)[6]$ & $1.49(0.59$ to 3.77$)[6]$ & $1.43(0.65$ to 3.15$)$ [9] & $1.45(0.74$ to 2.84$)[15]$ \\
\hline Unsteady/ataxia (A and B) & $5.21(2.09$ to 12.9$)[6]$ & $1.31(0.56$ to 3.05$)[7]$ & $1.31(0.64$ to 2.66$)[11]$ & $1.31(0.72$ to 2.39$)[18]$ \\
\hline Limping (D) & $1.02(0.45$ to 2.33$)[6]$ & $1.05(0.71$ to 1.57$)$ [30] & $0.75(0.51$ to 1.10$)[32]$ & $0.87(0.64$ to 1.17$)$ [62] \\
\hline
\end{tabular}




\begin{tabular}{|c|c|c|c|}
\hline (A) Type of gait disorder (category) & Three doses by 4 months of age & RI $(95 \% \mathrm{Cl})[\mathrm{n}]$ for 6-60 days & p value comparing No, Yes \\
\hline \multirow[t]{2}{*}{ All cases (A to F) } & No & $0.99(0.80$ to 1.23$)$ [117] & 0.27 \\
\hline & Yes & $0.82(0.60$ to 1.12$)$ [52] & \\
\hline \multirow[t]{2}{*}{ Unsteady gait (B) } & No & $1.59(0.70$ to 3.63$)[9]$ & 0.86 \\
\hline & Yes & $1.43(0.51$ to 4.02$)[6]$ & \\
\hline \multirow[t]{2}{*}{ Unsteady/ataxia (A and B) } & No & $1.41(0.68$ to 2.93 [11] & 0.84 \\
\hline & Yes & 1.34 (0.53 to 3.41$)[7]$ & \\
\hline (B) Type of gait disorder (category) & Mercury exposure intensity & RI $(95 \% \mathrm{Cl})[\mathrm{n}]$ for 6-60 days & p value comparing quartiles \\
\hline \multirow[t]{4}{*}{ All cases (A to F) } & Quartile 1 & $0.83(0.56$ to 1.22$)[31]$ & 0.76 \\
\hline & Quartile 2 & $1.04(0.76$ to 1.42$)[50]$ & \\
\hline & Quartile 3 & $0.99(0.72$ to 1.36$)$ [ 49$]$ & \\
\hline & Quartile 4 & 0.85 (0.60 to 1.21$)$ [39] & \\
\hline \multirow[t]{4}{*}{ Unsteady/ataxia (A and B) } & Quartile 1 & $1.30(0.35$ to 4.79$)[3]$ & 0.81 \\
\hline & Quartile 2 & $1.77(0.71$ to 4.42$)[7]$ & \\
\hline & Quartile 3 & $1.03(0.28$ to 3.80$)$ [3] & \\
\hline & Quartile 4 & $1.30(0.45$ to 3.81$)[5]$ & \\
\hline
\end{tabular}

with non-neurological reasons for the gait disturbance. In the hospital admission data set, clinical records were reviewed for $87 \%$ of cases, of which over half were considered to have a non-ataxic, non-viral episode of gait disturbance. However, there was still no evidence of a vaccine effect when separate analyses were performed, restricted to the more specific diagnostic codes. Of the 19 hospital admitted cases that were categorised as most similar to those described by Plesner ${ }^{1}$ (category 2), only two were in the putative post-vaccination risk period of 0-60 days after MMR vaccine (relative incidence $1.13,95 \%$ CI 0.21 to 6.20 ). While the confidence intervals around this estimate are fairly wide, the point estimate is close to one and provides no evidence in support of the view that MMR vaccine is associated with ataxia of unknown aetiology. Furthermore, when combined with the ataxia cases in the GPRD study the relative incidence for the 0-60 day period is 0.96 with a narrower $95 \%$ CI of 0.33 to 2.82 .

The high proportion (around two thirds) of cases in the GPRD dataset, where attempted validation was unsuccessful, was disappointing. This was largely due to the practice no longer participating in the GPRD, or the child no longer being registered with a participating practice. However, of the subset that could be validated, some had gait problems diagnosed on the day they received the MMR. Based on biological plausibility arguments, such cases were considered unlikely to be causally related to the vaccination, an assumption that was confirmed by the subset that could be validated. The effect of such cases was allowed for by removing the $0-5$ days post-vaccination period from the risk period. In the subsequent 6-60 day period there was no evidence of an increased risk of GP consultation for possible gait disturbance within two months of MMR vaccination. Furthermore, MMR vaccinated children with the highest levels of mercury exposure during the first 4-6 months of life were at no increased risk of gait disturbance.

Transient synovitis (including irritable hip) is a quite common but ill defined condition of uncertain aetiology which may follow a viral illness and present with gait disturbance. Rubella and occasionally rubella vaccine is a known cause of arthralgia/arthritis in older people, particularly women. MMR vaccination may occasionally be associated with joint or limb pain. ${ }^{10}{ }^{11}$ Our results showed no association between MMR vaccination and hospitalisation for transient synovitis.

"Gait disturbance" is a broad entity, encompassing a wide range of diagnostic possibilities, including trauma and drug ingestion as well as various neurological anomalies. A child with upper respiratory infection, particularly an infection associated with otitis media, may be transiently unsteady and appear ataxic. The loss of wellbeing associated with any viral infection may result in a toddler, particularly in the early weeks or months of ambulation, regressing transiently in his or her walking. Such "regression" may be interpreted as neurological abnormality. Viral infections occur frequently in early life and will occur coincidentally around the time of MMR vaccination. Although detailed validation proved not possible, the wide range of Read/OXMIS codes identified as gait disturbance in the general practice study group, undoubtedly included some of the above possibilities.

Plesner ${ }^{1}$ reported a type of gait disturbance consistent with cerebellar ataxia or other possible acute viral or post-viral encephalitis/cerebellitis, temporarily associated with MMR vaccination. However, follow up of 32 of the original 42 reported children, 1-10 years after the "acute" gait disturbance $^{12}$ showed some neurological and/or neuropsychological disorder in 15 of 26 children assessed; neuropsychological dysfunction was considered within the expected range for Danish children, but comparison data were not available for the minor motor disorders identified at follow up. The authors concluded that the cause of the children's apparent disorders at follow up could not be identified. It seems possible that the "acute" symptoms reported following MMR, in at least some of the children, were part of a pre-existing, and ongoing, mild neurodevelopmental disorder; nothing to do with the MMR vaccination. Compared with Plesner's reported rate of six cases of gait disturbance per 100000 MMR vaccinated children, our incidence rates were 4.1 per 100000 for hospital cases (category 2) and 17.4 for GPRD cases (category A, a less definite group than the well validated hospital cases.)

It has been suggested that the gait disturbance reported by Plesner $^{1}$ in temporal association with MMR, was due to the component viruses of the combined vaccine interacting in a novel way to cause subtle neurological effects not seen when the vaccines are given separately. ${ }^{13}$ The alleged association between MMR and gait disturbance has also been invoked to support the belief that MMR vaccine causes autism, as it is claimed that parents who suspect a causal link with the vaccine, frequently report gait disturbance in their previously normal children. ${ }^{13}$ Our study provides no evidence in support of these speculations and emphasises the importance of subjecting such claims to objective scientific scrutiny before 
invoking them in support of putative causal associations between MMR and various neurological conditions.

\section{ACKNOWLEDGEMENTS}

Support for the salary of J Stowe was provided by a grant from the Department of Health Research and Development Division (grant number 121/7470). The GPRD study was supported by a grant from the World Health Organisation to investigate the effect of thiomersal exposure on neurodevelopmental outcomes (grant number 18/181/ 854).

\section{Authors' affiliations}

E Miller, N Andrews, A Grant, Health Protection Agency, London, UK J Stowe, B Taylor, Royal Free Hospital, London, UK

Competing interests: none declared

\section{REFERENCES}

1 Plesner AM. Gait disturbance after MMR vaccine. Lancet 1995;345:316.

2 Miller E, Waight $P$, Farrington P. Safety assessment post-licensure. Dev Biol Stand 1998:95:235-43.

3 Farrington CP, Nash J, Miller E. Case-series analysis of adverse reactions to vaccines: a comparative study. Am J Epidemiol 1996;143:1165-73.
4 Wakefield AJ. Measles, mumps, and rubella vaccination and autism. N Engl J Med 2003;348:951-4.

5 Nash JQ, Chandrakumar $M$, Farrington $C P$, et al. Feasibility study for routine surveillance by record linkage of adverse events attributable to vaccination. Epidemiol Infect 1995;114:475-80.

6 Department of Health. Immunisation against infectious disease. London: HMSO, 1996:99, 105.

7 Andrews N, Miller E, Grant A, et al. Thiomersal exposure in infants and developmental disorders: a retrospective cohort study in the United Kingdom does not support a causal association. Submitted.

8 Peltola H, Heinonen OP. Frequency of true adverse reactions to measlesmumps-rubella vaccine. A double-blind placebo-controlled trial in twins. Lancet 1986; 1:939-42.

9 Department of Health. Government Statistical Service. NHS immunisation statistics, England: 2001-02. Statistical Bulletin 2002/18. September 2002 Available at http://www.doh.gov.uk/public/sb0218.pdf.

10 Virtanen $M$, Peltola $H$, Paunio $M$, et al. Day-to-day reactogenicity and the healthy vaccine effect of measles-mumps-rubella vaccination. Pediatrics 2000;106:e62.

11 Benjamin CM, Chew GC, Silman AJ. Joint and limb symptoms in children after immunisation with measles, mumps and rubella vaccine. $B M J$ 1992;304:1075-8

12 Plesner A-M, Hansen FJ, Taudort K, et al. Gait disturbance interpreted as cerebellar ataxia after MMR vaccination at 15 months of age: a follow-up study. Acta Paediatr 2000;89:58-63.

13 Wakefield AJ, Montgomery SM. Measles, mumps and rubella vaccine: through a glass darkly. Adverse Drug React Toxicol Rev 2000;19:265-83.

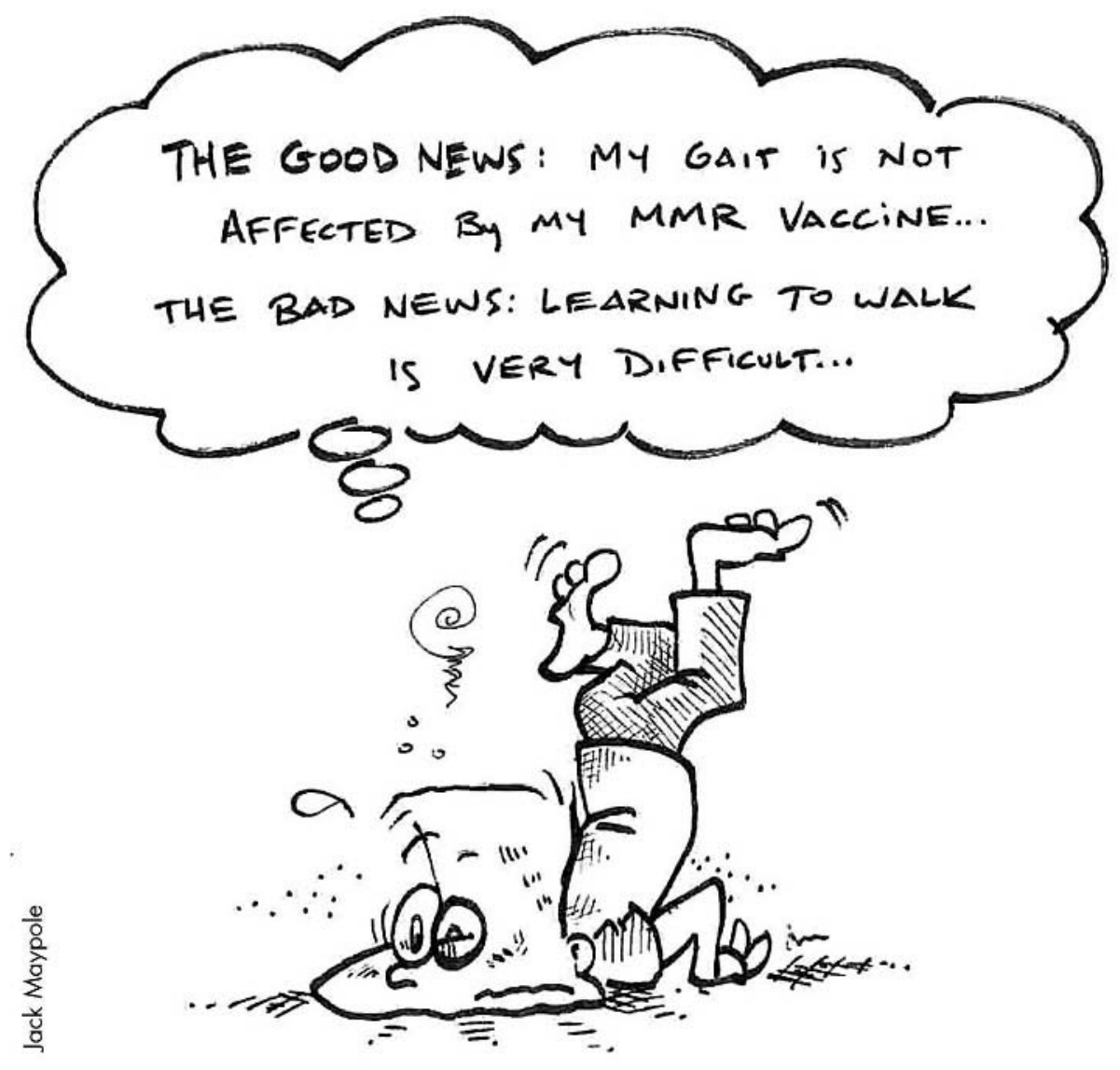

